\title{
Acute Pulmonary Embolism Is an Independent Predictor of Adverse Events in Severe Decompensated Heart Failure Patients*
}

\author{
Eduardo S. Darze, MD; Adriana L. Latado, MD, PhD; Aloyra G. Guimarães, MD; \\ Rodrigo A. V. Guedes, MD; Alessandra B. Santos, MD; Simone S. de Moura, MD; \\ and Luiz Carlos Santana Passos, MD, PhD
}

\begin{abstract}
Background: Congestive heart failure (CHF) is a well-recognized risk factor for venous thromboembolism (VTE) and is associated with higher mortality in patients with an acute pulmonary embolism (PE). There are very few data on how acute PE affects the clinical course of patients with heart failure. The purpose of this study was to determine the impact of an acute PE on the short-term prognosis of patients hospitalized for decompensated CHF.

Methods: This was a prospective cohort study of 198 patients admitted to a coronary care unit between July 2001 and March 2003 with severe decompensated CHF. The primary outcome measure was death or rehospitalization at 3 months.

Results: PE was confirmed in 18 of 198 patients enrolled (9.1\%). The groups with and without PE were comparable with regards to demographics, the prevalence of comorbid conditions, and severity of CHF ( $p>0.05)$. The prevalence of cancer $(p=0.0001)$, previous VTE $(p=0.003)$, and right ventricular overload $(p=0.006)$ was higher in the PE group. The presence of $\mathrm{PE}$ was also associated with a longer hospital stay $(37.5 \pm 71.6$ days vs $15.4 \pm 15.0$ days, $p=0.001)$ [mean \pm SD] and a higher incidence of death or rehospitalization at 3 months $(72.2 \%$ vs $43.9 \%, p=0.02)$. In a multiple logistic regression analysis, $\mathrm{PE}$ remained an independent predictor of death or rehospitalization at 3 months (odds ratio, $4.0 ; 95 \%$ confidence interval, 1.1 to $15.1 ; p=0.038$ ).

Conclusions: Acute PE commonly complicates the hospital course of patients with severe CHF, increasing the length of hospital stay and the chance of death or rehospitalization at 3 months.
\end{abstract}

(CHEST 2007; 131:1838-1843)

Key words: congestive heart failure; ICU; mortality; pulmonary embolism

Abbreviations: $\mathrm{CCU}=$ coronary care unit; $\mathrm{CHF}=$ congestive heart failure; $\mathrm{CI}=$ confidence interval; NYHA $=$ New York Heart Association; $\mathrm{OR}=$ odds ratio; $\mathrm{PE}=$ pulmonary embolism; VTE = venous thromboembolism

$\mathbf{V}$ enous thromboembolism (VTE) and congestive heart failure $(\mathrm{CHF})$ are among the most commonly encountered conditions in hospitalized patients, ${ }^{1,2}$ and each of them contribute to the significant morbidity and mortality observed in

*From the PhD Program (Dr. Darze) and Medicine Department (Dr. Passos), School of Medicine, Federal University of Bahia; and Coronary Care Unit (Drs. Latado, Guimarães, Guedes, Santos, and de Moura), Hospital Português, Salvador, Brazil.

This work was performed with no financial support at the Coronary Care Unit, Hospital Português, Salvador, Brazil.

None of the authors have any potential conflicts of interest to disclose. Manuscript received August 19, 2006; revision accepted February 13, 2007. this population. ${ }^{1,3,4}$ Heart failure is an important risk factor for VTE in ambulatory ${ }^{5}$ and hospitalized $^{6}$ patients, and a particularly high incidence of VTE has been documented in patients with severe decompensated CHF. ${ }^{7}$

Reproduction of this article is prohibited without written permission from the American College of Chest Physicians (www.chestjournal. org/misc/reprints.shtml).

Correspondence to: Eduardo S. Darze, MD, Programa de Pós-graduação em Medicina e Saúde, Universidade Federal da Bahia, Rua Padre Feijó, $2403^{\circ}$ andar, Ambulatório Magalhães Neto, Canela, 40110160 Salvador,Bahia,Brasil; e-mail: esdarze@ufba.br

DOI: 10.1378/chest.06-2077 
In the largest pulmonary embolism (PE) registry ${ }^{8}$ and in other cohort studies, ${ }^{9}$ the presence of $\mathrm{CHF}$ has been consistently associated with a higher mortality. In the International Cooperative Pulmonary Embolism Registry, 8 the prevalence of $\mathrm{CHF}$ was $11 \%$, and on multiple regression modeling it was found to be a significant prognostic factor, more than doubling the chance of death (hazard ratio, 2.4). The impact of PE, however, on the clinical course of CHF patients is not known. The goal of the current study was to address this issue in a population of hospitalized patients with severe heart failure.

\section{Materials AND Methods}

\section{Patient Population}

We prospectively enrolled consecutive patients admitted to our coronary care unit (CCU) with the diagnosis of CHF between July 2001 and March 2003. After the evaluation of the on-call physician, two investigators reviewed the clinical data in order to ascertain the diagnosis of $\mathrm{CHF}$ in patients before entering the study. Patients were excluded if they had an acute ST-segment elevation myocardial infarction and if they had a different admitting diagnosis despite having a history of CHF. All clinical and demographic data were collected directly from the patient, family member, attending physician, or chart. The enrolled patients were followed on a daily basis until hospital discharge. Three months after enrollment, the patients were contacted by telephone in order to obtain information regarding the combined end-point of death or rehospitalization. This study complies with the Declaration of Helsinki, was approved by the research ethics committee, and written informed consent was obtained from all patients.

\section{Definitions}

Confirmed PE was defined as any level of clinical suspicion associated with the following: (1) high-probability lung scintigraphy ${ }^{10}$ (two or more large segmental mismatches, or one large plus two or more moderate mismatches, or four or more moderate mismatches); or (2) positive spiral CT of the chest ${ }^{11}$ (presence of a hypoattenuating intraluminal filling defect in a pulmonary artery); or (3) positive conventional pulmonary angiography. Investigation for $\mathrm{PE}$ was initiated at the discretion of the attending physician. A total of 36 patients were investigated, and 18 had the diagnosis of PE confirmed. The other 18 patients had non-high-probability lung scans or negative chest CT findings. The only other imaging modality used in these patients was Doppler ultrasonography, and none had confirmed deep vein thrombosis. These patients were included in the group without $\mathrm{PE}$ and were observed for 3 months.

Cancer was considered to be present only if it had been treated within the last 6 months, or the patient was receiving palliative care. Acute renal failure was defined as an elevation in the creatinine concentration during hospitalization to $\geq 1.5 \mathrm{mg} / \mathrm{dL}$ with previously normal creatinine levels, or a $25 \%$ increase compared to the baseline values. Anemia was defined according to the World Health Organization criteria: hemoglobin $<12.0$ $\mathrm{g} / \mathrm{dL}$ for female patients and $<13.0 \mathrm{~g} / \mathrm{dL}$ for male patients, and hyponatremia defined as a sodium level $<136 \mathrm{mEq} / \mathrm{L}$. The New York Heart Association (NYHA) functional class referred to the patient status 3 weeks prior to the index admission.

\section{Statistical Analysis}

The comparisons between the groups with and without PE were performed using the unpaired Student $t$ test for continuous variables and $\chi^{2}$ test for categorical variables. All values were expressed as mean \pm SD or as frequency (\%).

A multiple logistic regression analysis was conducted in order to identify independent predictors of the primary combined end point of death or rehospitalization at 3 months. The secondary end points were death at 3 months, rehospitalization at 3 months, and length of hospital stay. All clinical end points were defined prospectively. In case both end points or repeated rehospitalizations were present, only one end point was counted. Continuous variables were categorized at clinically relevant cut points. Variables with significant univariate associations with the combined end point $(\mathrm{p}<0.1)$ or variables thought to be clinically important were included in a multivariate model. A $p$ value $<0.05$ was considered significant. Statistical analysis was performed using statistical software (SPSS for Windows version 10; SPSS; Chicago, IL).

\section{RESULTS}

During the study period, 223 patients were admitted to the CCU with a diagnosis of CHF. A total of 25 patients were excluded for the following reasons: acute ST-segment elevation myocardial infarction $(\mathrm{n}=14)$, and unconfirmed diagnosis of CHF $(\mathrm{n}=11)$ The study population was comprised of 198 patients, and general characteristics of the population according to the presence of $\mathrm{PE}$ are presented on Table 1. Mean age $(68.2 \pm 14.1$ years vs $69.6 \pm 13.4$ years, $\mathrm{p}=0.67)$ and the prevalence of male gender $(61.1 \%$ vs $55.0 \%, \mathrm{p}=0.62)$ were similar in the groups with and without $\mathrm{PE}$, as was the prevalence of comorbid conditions. Overall, this was a population with severe CHF as demonstrated by a high prevalence of severe left ventricular dysfunction (38.9\% vs $42.1 \%$; $p=0.79)$, poor functional class at baseline (NYHA class III/IV, $55.6 \%$ vs $55.0 \%$; $\mathrm{p}=0.96), \quad$ hyponatremia $(33.3 \%$ vs $21.1 \%$; $\mathrm{p}=0.23)$, and prior hospitalizations for $\mathrm{CHF}(64.7 \%$ vs $71.2 \% ; \mathrm{p}=0.56)$. The prevalence of risk factors for VTE such as cancer $(\mathrm{p}=0.0001)$, previous VTE $(\mathrm{p}=0.003)$, and immobilization $(\mathrm{p}=0.018)$ was higher in the PE group. Right ventricular overload was also significantly more frequent in the PE group ( $\mathrm{p}=0.006)$.

The diagnosis of $\mathrm{PE}$ was confirmed in 18 of 198 patients $(9.1 \%)$ during their hospitalization period. The diagnostic methods utilized were lung scintigraphy in 14 patients (78\%) and spiral CT in 4 patients $(22 \%)$. Doppler ultrasonography demonstrated deep vein thrombosis in 8 of 18 patients (44.4\%) with PE: seven cases involving the lower extremities, and one subclavian vein thrombosis. The majority of $\mathrm{PE}$ patients had their diagnosis confirmed within 5 days of hospital admission (67\%). After the diagnosis, all patients received anticoagulation with therapeutic 
Table 1-Patient Characteristics According to the Presence of PE*

\begin{tabular}{|c|c|c|c|}
\hline Characteristics & $\mathrm{PE}(\mathrm{n}=18)$ & No PE $(\mathrm{n}=180)$ & p Value \\
\hline Age, yr & $68.2(14.1)$ & $69.6(13.4)$ & 0.67 \\
\hline Male gender & 61.1 & 55.0 & 0.62 \\
\hline \multicolumn{4}{|l|}{ Comorbid conditions } \\
\hline Diabetes & 33.3 & 33.9 & 0.96 \\
\hline Hypertension & 66.7 & 79.8 & 0.20 \\
\hline Chronic renal failure & 33.3 & 31.1 & 0.85 \\
\hline Anemiał & 61.1 & 50.6 & 0.39 \\
\hline Cerebrovascular disease & 16.7 & 15.0 & 0.85 \\
\hline Atrial fibrillation & 16.7 & 26.0 & 0.39 \\
\hline COPD & 16.7 & 5.0 & 0.05 \\
\hline Smoking & 38.9 & 28.8 & 0.37 \\
\hline \multicolumn{4}{|l|}{ CHF severity } \\
\hline Ischemic etiology & 55.6 & 53.9 & 0.89 \\
\hline Ejection fraction $<30 \%$ & 38.9 & 42.1 & 0.79 \\
\hline Restrictive pattern on mitral inflow & 33.3 & 28.7 & 0.68 \\
\hline NYHA functional class III-IV $+\underset{+}{+}$ & 55.6 & 55.0 & 0.96 \\
\hline $\mathrm{Na}<136 \mathrm{mEq} / \mathrm{L}$ & 33.3 & 21.1 & 0.23 \\
\hline Previous hospitalization for $\mathrm{CHF}$ & 64.7 & 71.2 & 0.56 \\
\hline Right ventricular overload $\$$ & 61.1 & 29.1 & 0.006 \\
\hline \multicolumn{4}{|l|}{ VTE risk factors } \\
\hline Cancer\| & 33.3 & 2.8 & $<0.0001$ \\
\hline Previous VTE & 22.2 & 4.5 & 0.003 \\
\hline Obesity (body mass index $>30 \mathrm{~kg} / \mathrm{m}^{2}$ ) & 5.6 & 13.6 & 0.33 \\
\hline Surgery within 1 mo & 5.6 & 1.7 & 0.27 \\
\hline Immobilization $\llbracket$ & 16.7 & 2.2 & 0.018 \\
\hline VTE prophylaxis (enoxaparin, 40 mg/d) & 66.7 & 70.0 & 0.77 \\
\hline \multicolumn{4}{|l|}{ In-hospital clinical events } \\
\hline Acute renal failure\# & 55.6 & 44.45 & 0.37 \\
\hline Hospital death & 33.3 & 21.7 & 0.26 \\
\hline Length of hospitalization, $\mathrm{d}$ & $37.5(71.6)$ & $15.4(15.0)$ & 0.001 \\
\hline
\end{tabular}

* Data are presented as mean (SD) or \%.

$\nmid$ Hemoglobin $<12.0 \mathrm{~g} / \mathrm{dL}$ for female patients and $<13.0 \mathrm{~g} / \mathrm{dL}$ for male patients.

\$Three weeks prior to hospital admission.

\$Echocardiographically documented right ventricular dilatation and/or systolic dysfunction.

||Treated within 6 months or receiving palliative care.

INot able to ambulate during the hospitalization period.

\#Creatinine levels $>1.5 \mathrm{mg} / \mathrm{dL}$ or a $25 \%$ increase compared to the baseline values.

doses of heparin, and one patient received an inferior vena cava filter. No patients received thrombolytic therapy.

The rates of clinical events according to the presence of PE are shown in Table 1 and Figure 1. At 3 months, the PE group presented a higher mortality rate $(50.0 \%$ [95\% confidence interval (CI), 26.8 to $73.2 \%$ ] vs $30.6 \%$ [95\% CI, 24.0 to $37.9 \%$, $\mathrm{p}=0.09)$ and rehospitalization rates $(58.3 \%[95 \%$ CI, 28.6 to $83.5 \%$ ] vs $24.1 \%$ [ $95 \%$ CI, 17.5 to $32.2 \%$ ], $\mathrm{p}=0.01)$. The incidence of the combined end point of death or rehospitalization at 3 months was significantly higher in the group with PE $(72.2 \%$ [95\% CI, 46.4 to $89.3 \%$ ] vs $43.9 \%$ [95\% CI, 36.6 to $51.5 \%$ ], $\mathrm{p}=0.02$ ). The mean length of hospitalization was $37.5 \pm 71.6$ days in the PE group and $15.4 \pm 15.0$ days in the group without PE ( $p=0.001$ ).

Table 2 shows the results of the univariate analysis for potential predictors of the combined end point of death and rehospitalization at 3 months. The presence of PE was significantly associated with the combined end point, and remained an independent predictor in a multiple logistic regression analysis (odds ratio [OR], 4.0; 95\% CI, 1.1 to $15.1 ; \mathrm{p}=0.038$ ) [Table 3]. Other significant predictors of the combined end point were cerebrovascular disease $(\mathrm{p}=0.004)$, prior hospitalization for $\mathrm{CHF}$ $(\mathrm{p}=0.002)$, hyponatremia $(\mathrm{p}=0.002)$, and acute renal failure $(\mathrm{p}=0.003)$.

\section{Discussion}

To the best of our knowledge, this is the first study to demonstrate that acute PE adversely affects the short-term clinical course of hospitalized patients with CHF, increasing the length of hospital stay and the chance of the combined end point of death or rehospitalization at 3 months. 


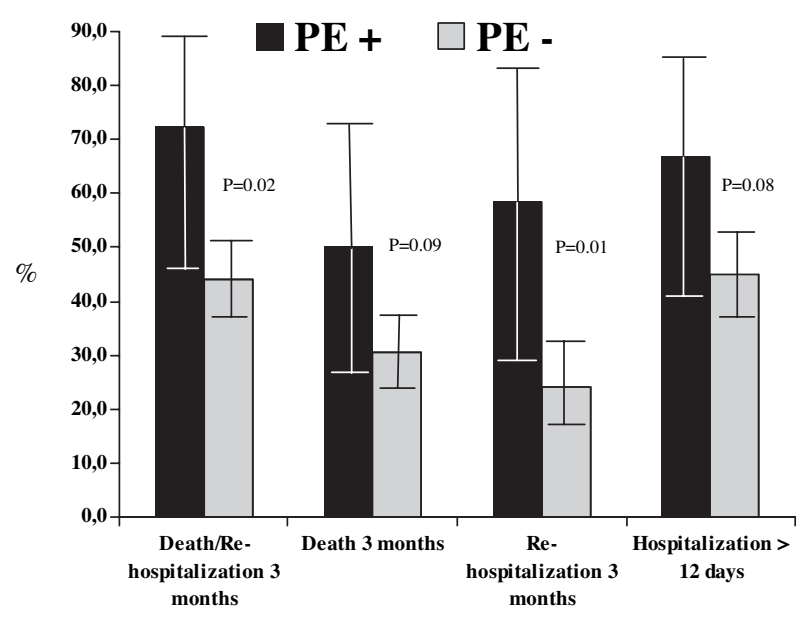

Figure 1. Clinical events according to the presence of PE.

The interaction between CHF and VTE is bidirectional and has multiple facets. With 550,000 new cases a year only in the United States, CHF is the most common admitting diagnosis in patients $>60$ years old. ${ }^{1}$ The disease process induces a number of rheologic alterations that lead to a chronic hypercoagulable state, which in association with venous stasis and reduced mobility places the patients at increased risk for VTE. ${ }^{12}$ The elevated risk of VTE in CHF patients has been confirmed in clinical ${ }^{7}$ and autopsy

Table 2-Univariate Predictors of Death or Rehospitalization at 3 Months

\begin{tabular}{|c|c|c|}
\hline Variables & OR $(95 \% \mathrm{CI})$ & p Value \\
\hline Age $>70 \mathrm{yr}$ & $1.62(0.92-2.85)$ & 0.097 \\
\hline Male gender & $1.17(0.67-2.05)$ & 0.598 \\
\hline Aspirin use & $0.43(0.24-0.76)$ & 0.0004 \\
\hline \multicolumn{3}{|l|}{ Comorbid conditions } \\
\hline Hypertension & $0.70(0.35-1.37)$ & 0.298 \\
\hline Diabetes & $1.30(0.72-2.34)$ & 0.388 \\
\hline COPD & $3.71(0.98-14.15)$ & 0.054 \\
\hline Anemia & $1.87(1.06-3.29)$ & 0.031 \\
\hline Cerebrovascular disease & $3.85(1.62-9.15)$ & 0.002 \\
\hline Chronic renal failure & $1.80(0.98-3.30)$ & 0.058 \\
\hline Atrial fibrillation & $1.37(0.72-2.62)$ & 0.341 \\
\hline Smoking & $1.43(0.80-2.73)$ & 0.218 \\
\hline Cancer & $1.40(0.42-4.79)$ & 0.580 \\
\hline \multicolumn{3}{|l|}{ CHF severity } \\
\hline NYHA functional class III/IV & $1.56(0.88-2.74)$ & 0.126 \\
\hline Ejection fraction $<30 \%$ & $1.28(0.72-2.26)$ & 0.395 \\
\hline $\begin{array}{l}\text { Restrictive pattern on mitral } \\
\text { inflow }\end{array}$ & $1.69(0.90-3.19)$ & 0.103 \\
\hline Ischemic etiology & $1.21(0.69-2.11)$ & 0.514 \\
\hline Hyponatremia (Na < 136 mEq/L) & $3.60(1.76-7.49)$ & $<0.0001$ \\
\hline Previous hospitalization & $3.49(1.77-6.88)$ & $<0.0001$ \\
\hline \multicolumn{3}{|l|}{ In-hospital complications } \\
\hline $\mathrm{PE}$ & $3.32(1.14-9.78)$ & 0.028 \\
\hline Acute renal failure & $3.02(1.69-5.39)$ & $<0.0001$ \\
\hline Length of hospitalization $>12 \mathrm{~d}$ & $2.44(1.38-4.33)$ & 0.002 \\
\hline
\end{tabular}

Table 3-Multiple Logistic Regression Analysis*

\begin{tabular}{lllc}
\hline \hline \multicolumn{1}{c}{ Variables } & OR & 95\% CI & p Value \\
\hline Cerebrovascular disease & 4.1 & $1.6-10.9$ & 0.004 \\
PE & 4.0 & $1.1-15.1$ & 0.038 \\
Hyponatremia & 3.7 & $1.6-8.5$ & 0.002 \\
Previous hospitalization & 3.4 & $1.6-7.6$ & 0.002 \\
Acute renal failure & 2.7 & $1.4-5.3$ & 0.003 \\
COPD & 4.0 & $0.9-18.1$ & 0.075 \\
\hline
\end{tabular}

*Hosmer-Lemenshow, $\mathrm{p}=0.593$.

studies. ${ }^{13}$ Additionally, it is also known that CHF is an independent predictor of mortality in patients with $\mathrm{PE}$, as shown in large cohort studies. ${ }^{8,9}$

However, the impact of a pulmonary thromboembolic event on the clinical course of CHF patients is not known. Probably due to diagnostic difficulties and low incidence of $\mathrm{PE}$, the large CHF series never addressed this issue. In a previous publication, ${ }^{7}$ we documented a very high incidence of $\mathrm{PE}$ in patients with severe decompensated $\mathrm{CHF}$, and the current study is the result of the 3-month clinical follow-up of the same population. The mean number of days spent at the hospital was two times greater in the patients with PE. Since 67\% of PE patients had their diagnosis confirmed within 5 days of hospital admission, PE was probably the cause rather than a consequence of the longer hospitalization period. We have also shown that even after adjustment for known prognostic variables, $\mathrm{PE}$ remained an independent predictor of clinical outcome. Specifically, COPD was significantly associated with the presence of PE and clinical outcomes. Nonetheless, PE maintained its association with a worse clinical outcome even after adjustment for the presence of COPD.

The understanding of the pathophysiologic events following an acute PE helps us explain its negative impact on the clinical course of CHF patients. The sudden increase in pulmonary pressures has multiple effects on right ventricle and left ventricle physiology. Initially, it results in right ventricular dilatation and dysfunction, leading to a reduction in cardiac output. ${ }^{14}$ Left ventricular filling is also decreased as a consequence of leftward shift of the interventricular septum and pericardial constrain. ${ }^{15}$ Systemic hypotension ensues leading to impaired coronary perfusion, which in association with the increase in wall stress and oxygen demand results in right ventricular ischemia and infarction. ${ }^{16,17}$ This vicious circle may ultimately lead to cardiogenic shock and death. The severity of this pathophysiologic sequence depends mainly on the degree of pulmonary vascular obstruction and the patient's cardiopulmonary reserve. ${ }^{18}$ Thus, in patients with CHF, especially those with coronary disease, even small pulmo- 
nary emboli may lead to important hemodynamic consequences, ${ }^{19,20}$ potentially triggering episodes of acute decompensation and some cases of sudden cardiac death.

A retrospective analysis of the Studies of Left Ventricular Dysfunction ${ }^{21}$ prevention and treatment database revealed that PE represented $16 \%$ of all thromboembolic events, $38 \%$ of which were fatal. This is a much higher mortality rate when compared to that observed in the largest international $\mathrm{PE}$ registry $(17.8 \%) .{ }^{8}$ In an interesting study ${ }^{22}$ consisting of $119 \mathrm{CHF}$ patients who died after having an implantable cardioverter defibrillator placed, PE was the cause of death in 5 patients $(4.2 \%)$. It is worth noting that the final rhythms were most commonly electromechanical dissociation and bradyarrhythmias, rhythms compatible with PE. Thus, this probably represents an underestimation of the real incidence of fatal PE. In another study ${ }^{23}$ involving 62 pediatric patients with end-stage dilated cardiomyopathy awaiting transplant, PE developed in 6 patients and 4 died within 6 weeks of anticoagulation therapy.

The demonstration of a higher mortality and rehospitalization rate in $\mathrm{CHF}$ patients with $\mathrm{PE}$ has important clinical implications. First, given its high incidence, physicians should maintain a high index of suspicion for $\mathrm{PE}$ in hospitalized $\mathrm{CHF}$ patients with an unexplained exacerbation because early diagnosis and treatment may improve clinical outcomes. In the current study, $50 \%$ of patients in whom $\mathrm{PE}$ was suspected had their diagnosis confirmed, which is a much higher proportion compared to other series, ${ }^{10}$ and confirms the high thromboembolic risk in this population. In fact, a major problem with the diagnosis of $\mathrm{PE}$ is the relatively low sensitivity of its diagnostic modalities. ${ }^{10}$ Therefore, it is possible that some of our CHF patients may have had undiagnosed PE, which only reinforces the conclusion of this study that $\mathrm{PE}$ is a common and dangerous complication in hospitalized $\mathrm{CHF}$ patients.

Second, although long-term anticoagulation has never been shown to benefit CHF patients in sinus rhythm, additional studies are needed to evaluate the effect of treatment in a specific population of $\mathrm{CHF}$ patients at high risk for PE. We have identified important predictors of $\mathrm{PE}$ in this population, such as right ventricular dilatation and/or dysfunction, cancer, and previous VTE. ${ }^{7}$

Two obvious limitations of this study should be emphasized. Recruitment was conducted at a single center, and the population studied included a specific group of patients with severe decompensated CHF admitted to a CCU. Both facts limit the generalization of our results to a wider-ranging $\mathrm{CHF}$ population. Additionally, as an observational study, we did not have control over which patients were selected for a PE workup or the interpretation of their diagnostic tests. The diagnosis of $\mathrm{PE}$ was made using well-established published criteria, ${ }^{10,11}$ and almost half of our PE patients had associated deep vein thrombosis. Given the high positive predictive value of the criteria utilized, ${ }^{10,11}$ the only potential diagnostic problem would be underdiagnosing PE. As discussed above, this would only serve to strengthen our conclusions. We also believe that the potential biased selection of patients for a PE workup did not affect our results in any meaningful way because the groups with and without PE were comparable (Table 1), and any potential confounding was accounted for in the multivariate analysis.

Our study adds to the literature by demonstrating that $\mathrm{PE}$ is frequently encountered and adversely affects the clinical course of patients with severe CHF. Further studies are necessary to assess the value of routine testing for $\mathrm{PE}$ in patients with $\mathrm{CHF}$ exacerbations, and to define the role of long-term anticoagulation for the prevention of thromboembolic events in this high-risk population.

\section{REFERENCES}

1 Heart disease and stroke statistics, 2003 update. Dallas, TX: American Heart Association, 2002

2 Samama MM, Cohen AT, Darmon J-Y, et al. A comparison of enoxaparin with placebo for the prevention of VTE in acutely ill medical patients. N Engl J Med 1999; 341:793-800

3 Rubinstein I, Murray D, Hoffstein V. Fatal pulmonary emboli in hospitalized patients: an autopsy study. Arch Intern Med 1988; 148:1425-1426

4 Baglin TP, White K, Charles A. Fatal pulmonary embolism in hospitalized medical patients. J Clin Pathol 1997; 50:609-618

5 Howell MD, Geraci JM, Knowlton AA. Congestive heart failure and outpatient risk of venous thromboembolism: a retrospective case-control study. J Clin Epidemiol 2001; 54:810-816

6 Alikhan R, Cohen AT, Combe S, et al. Risk factors for venous thromboembolism in hospitalized patients with acute medical illness. Arch Intern Med 2004; 164:963-968

7 Darze ES, Latado AL, Guimarães AG, et al. Incidence and clinical predictors of pulmonary embolism in severe heart failure patients admitted to a coronary care unit. Chest 2005; 128:2576-2580

8 Goldhaber SZ, Visani L, De Rosa M, for ICOPER. Acute pulmonary embolism: clinical outcomes in the International Cooperative Pulmonary Embolism Registry. Lancet 1999; 353:1386-1389

9 Heit JA, Silverstein MD, Mohr DN, et al. Predictors of survival after deep vein thrombosis and pulmonary embolism: a population based cohort study. Arch Intern Med 1999; 159:445-453

10 Freitas JE, Sarosi MG, Nagle CC, et al. Modified PIOPED criteria used in clinical practice. J Nucl Med 1995; 36:15731578

11 Remy-Jardin M, Remy J, Wattinne L, et al. Central pulmonary thromboembolism: diagnosis with spiral volumetric CT with the single-breath-hold technique; comparison with pulmonary angiography. Radiology 1992; 185:381-387 
12 Gibbs CR, Blann AD, Watson RDS, et al. Abnormalities of hemorheological, endothelial, and platelet function in patients with chronic heart failure in sinus rhythm. Circulation 2001; 103:1746-1751

13 Roberts WC, Siegel RJ, McManus BM. Idiopathic dilated cardiomyopathy: analysis of 152 necropsy patients. Am J Cardiol 1987; 60:1340-1355

14 Tachini AC, Fermoso JD, Aramendia P. Behavior of the right ventricle following acute constriction of the pulmonary artery. Circ Res 1960; 8:315-318

15 Jardin F, Dubourg O, Gueret P, et al. Quantitative twodimensional echocardiography in massive pulmonary embolism: emphasis on ventricular interdependence and leftward septal displacement. J Am Coll Cardiol 1987; 10:1201-1206

16 Vlahakes GL, Turley K, Hoffman JIE. The pathophysiology of failure in acute right ventricular hypertension: hemodynamic and biochemical correlations. Circulation 1981; 63:87-95

17 Adams JE, Siegel BA, Goldstein JA, et al. Elevations of $\mathrm{CK}-\mathrm{MB}$ following pulmonary embolism: a manifestation of occult right ventricular infarction. Chest 1992; 101:12031206
18 McIntyre KM, Sasahara AA. Determinants of right ventricular function and hemodynamics after pulmonary embolism. Chest 1974; 65:534-543

19 UKEP Study Research Group. The UKEP Study: multicentre clinical trial on two local regimens of urokinase in massive pulmonary embolism. Eur Heart J 1987; 8:2-10

20 Brooks H, Kirk ES, Vokonas PS, et al. Performance of the right ventricle under stress: relation to right coronary flow. J Clin Invest 1971; 50:2176-2183

21 Dries DL, Rosenberg YD, Waclawiw MA, et al. Ejection fraction and risk of thromboembolic events in patients with systolic dysfunction and sinus rhythm: evidence for gender differences in the studies of left ventricular dysfunction trials. J Am Coll Cardiol 1997; 29:1074-1080

22 Grubman EM, Pavri BB, Shipman T, et al. Cardiac death and stored electrograms in patients with third-generation implantable cardioverter-defibrillators. J Am Coll Cardiol 1998; 32:1056-1062

23 Hsu DT, Addonizio LJ, Hordof AJ, et al. Acute pulmonary embolism in pediatric patients awaiting heart transplantation. J Am Coll Cardiol 1991; 17:1621-1625 